\title{
Dancing eyes and dancing feet syndrome
}

\author{
${ }^{1} \mathrm{P}$ Manouri Senanayake and ${ }^{2} \mathrm{SP}$ Sumanasena
}

(Index words: Ganglioneuroblastoma, opsoclonus-myoclonus syndrome, surgery)

\section{Introduction}

Opsoclonus-myoclonus is a rare neurological disorder characterised by intention tremor, unsteady gait, myoclonus and opsoclonus. Lethargy and irritability are also described. The abnormal eye movements are distinctive, being multidirectional and of high amplitude. Its recognition helps in the diagnosis of this clinical syndrome, the correct identification of which leads to the detection of an underlying neuroblastoma or other disorders.

\section{Case report}

An 18-month old, previously healthy girl presented with progressively worsening unsteady gait of 6 weeks' duration. There was no preceding viral infection. On examination she was irritable, tremulous and unable to stand or walk without help. She had a staggering gait, continuous myoclonus of neck and limbs, and difficulty in speaking and swallowing. She had rapid, irregular, oscillatory eye movements. Cranial nerves, optic fundi, muscle power, tone, reflexes and sensation were normal.

Blood pressure was within the normal range for her age and the rest of the physical examination, including abdomen, was normal. Her growth curve showed flattening over the past 3 months.

Investigations with EEG and CT scan of the brain earlier had not shown any abnormality. A calcified suprarenal mass $(2.9 \times 2.5 \times 2.8 \mathrm{~cm})$ encasing the inferior vena cava on the right side, and enlarged para-aortic lymph nodes were found on ultrasound examination and were confirmed by computerised axial tomography (Figure 1). Bone marrow examination was normal.

The abdominal tumour and lymph nodes were surgically excised. Histopathological diagnosis was a ganglioneuroblastoma arising from the sympathetic chain and secondary deposits in lymph nodes. Surgery and chemotherapy were followed by marked regression of the neurological symptoms.

\section{Discussion}

The syndrome of opsoclonus, myoclonus, ataxia and encephalopathy has been termed "Kinsbourne syndrome", "dancing eyes and dancing feet syndrome" and "opsoclonusmyoclonus syndrome" [1]. When they occur separately, opsoclonus and myoclonus have many causes, but when in combination, the cause is either post-viral $(50 \%)$, or associated with an underlying tumour (50\%).

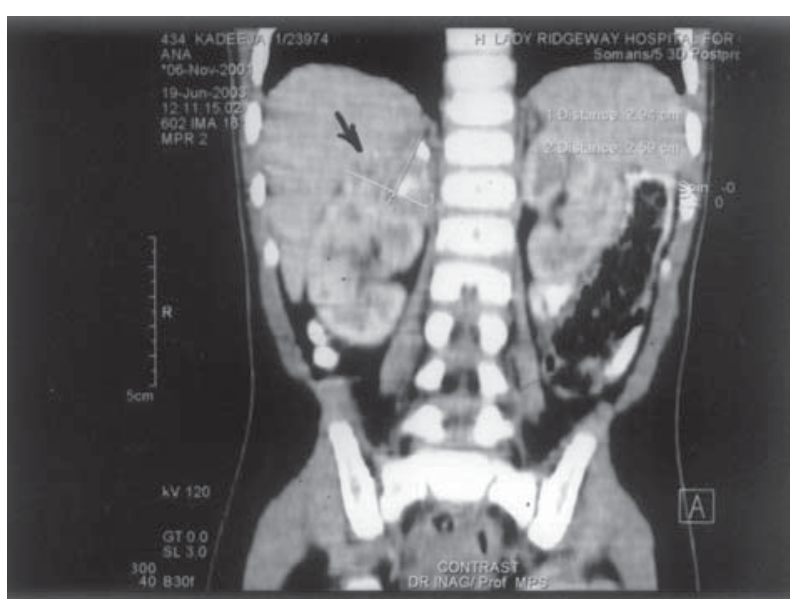

Figure 1. CT Scan of abdomen showing adrenal mass and enlarged para-aortic lymph nodes.

Epstein Barr, Coxsackie B, mumps, polio and other enteroviruses are among the virus infections documented to precede this syndrome [2]. However, the search for a tumour should not be prevented by evidence of a viral infection. In children the syndrome usually presents in the first 2 years. Both sexes are equally affected. Tumours that cause opsoclonus-myoclonus are those arising from "neural crest cells", i.e. neuroblastoma or ganglioneuroblastoma $[3,4]$.

An autoimmune basis is the likely explanation for this syndrome, because the brain and tumours of neural crest origin share the same embryonic forerunners, and have similar antigenic properties. The brain is therefore, "an innocent bystander caught in the cross-fires" between the immune system and the tumour. Facilities to test for anti$\mathrm{Hu}$ antibodies in the serum were unavailable to us [5].

Removal of the tumour does not always result in complete regression of the neurological symptoms. Other treatment options include ACTH or steroid therapy, intravenous human immunoglobulin therapy, or azathioprine. Better survival rates have been reported in children who manifest opsoclonus-myoclonus [6, 7]. Awareness of this uncommon clinical entity is important for early recognition and prognosis of neuroblastoma, the commonest extracranial solid tumour of childhood.

${ }^{1}$ Professor and Head, and 'Lecturer, Department of Paediatrics, Faculty of Medicine, Kynsey Road, Colombo 8. E-mail: <paeds@sltnet.lk> (Competing interests: none declared). Received 15 December 2003 and revised version accepted 20 February 2004. 


\section{Case reports}

\section{References}

1. Kinsbourne M. Myoclonic encephalopathy of infancy. Journal of Neurology Neurosurgery and Psychiatry 1962, 25: 271-6.

2. Komal E Imitiaz, Vora JP. Dancing eyes-dancing feet. Lancet 1999; 354: 390.

3. Shapiro B, Shulkin B, Hutchinson R, Bass J, Gross N, et al. Locating neuroblastoma in the opsoclonus myoclonus syndrome. Journal of Nuclear Biology and Medicine 1994, 38: $545-55$.

4. Storey I, Denniston A, Denniston S. Dancing eyes. Hospital Medicine 2003; 64: 555-6.
5. Posner JB. Autoantibodies in childhood opsoclonusmyoclonus syndrome. Journal of Pediatrics 1997, 130: 855.

6. Altman AJ, Baehner RL. Favourable prognosis for survival in children with coincident opso-myoclonus and neuroblastoma. Cancer 1976, 37: 846-52.

7. Koh PS, Raffensperger JG, Berry S, Larsen $M B$, Johnstone, MS, et al. Long-term outcome in children with opsoclonus-myoclonus and ataxia and coincident neuroblastoma. Journal of Pediatrics 1994, 125: 712-6. 